1 Hacettepe Journal of Mathematics and Statistics

$\bigcap$ Volume 45 (4) (2016), $1007-1014$

\title{
Spectral singularities of the matrix Schrödinger equations
}

\author{
Elgiz Bairamov* and Şerifenur Cebesoy ${ }^{\dagger}$
}

\begin{abstract}
In this paper, we investigate analytical and asymptotical properties of the Jost function of the matrix Schrödinger equation. Later, using the analytic continuation and the uniqueness theorems of analytic functions we study the eigenvalues and the spectral singularities of that equation.
\end{abstract}

Keywords: Differential Equations, Jost Function, Eigenvalues, Spectral Singularities

2000 AMS Classification: 34B05, 34B27, 34L05

Received : 19.06.2015 Accepted : 05.10.2015 Doi : 10.15672/HJMS.20164514275

\section{Introduction}

Schrödinger differential equations subject to the general point interaction can be found in many real world problems. These equations describe observed evolution phenomena. For instance, many chemical, physical phenomena and pharmacokinetics do exhibit point interaction effects [1]. The spectral analysis of Schrödinger equations with general point interaction have been investigated in detail in [2]-[6]. To be more precise, we should note that these equations have bound states, i.e., eigenvalues with square-integrable eigenfunctions and spectral singularities. It is well known that the bound state of quantum mechanical system correspond to the energy. Also a physical interpretation for the spectral singularities that identifies with the energies of scattering states having infinite reflection and transmission

coefficients. So spectral singularities correspond to the resonance states having a real energy. Consequently, the spectral analysis of Schrödinger equations with

spectral singularities are important to study in quantum mechanics. So in this paper, we investigate the spectral analysis of general matrix Schrödinger equations with spectral singularities.

*Department of Mathematics, Ankara University, 06100 Tandoğan, Ankara, TURKEY

Email : bairamov@science.ankara.edu.tr

${ }^{\dagger}$ Department of Mathematics, Ankara University, 06100 Tandoğan, Ankara, TURKEY

Email : scebes oy@ankara.edu.tr 
The spectral analysis of differential equations with spectral singularities was investigated by Naimark [7]. Schwartz studied the spectral singularities of a certain class of abstract linear operators in a Hilbert space [8]. The following definition of spectral singularities is given by Schwartz.

Let $H$ be a Hilbert space and $A: H \rightarrow H$ be a linear operator such that its spectrum $\sigma(A)$ consists of an interval $J$ of the real axis and a finite number of complex numbers outside $J$. We will denote the resolvent operator of $A$ by $R_{\mu}(A):=(A-\mu I)^{-1}$, where $\mu \in \mathbb{C}$. Let $J_{0}$ be a finite subset of $J$. Assume that for any finite subinterval $\Delta$ of $J$, whose closure do not contain any point of $J_{0}$, the limit operator

$$
E_{\Delta}=\lim _{\epsilon \rightarrow 0^{+}} \frac{1}{2 \pi i} \int_{\Delta}\left[R_{\mu+i \epsilon}(A)-R_{\mu-i \epsilon}(A)\right] d \mu
$$

exists in the strong limit sense, so that $E_{\Delta}$ is a linear bounded operator on $H$. Denote by $d$ the distance from the interval $\Delta$ to the set $J_{0}$. If

$$
\lim _{d \rightarrow 0}\left\|E_{\Delta}\right\|=\infty
$$

then any point of the set $J_{0}$, is called a spectral singularity of the operator $A$. For the selfadjoint operators $\left\|E_{\Delta}\right\| \leq 1$, so that selfadjoint operators have no spectral singularities.

The sets of the spectral singularities for closed linear operators on a Banach space was given by Nagy [9]. Nagy shows that the set of spectral singularities defined according to his general definition coincides in the case of differential operators as defined by Naimark and Lyance [7], [10]. Pavlov established the dependence of the structure of the spectral singularities of Schrödinger operators on the behaviour of the potential function at infinity [11].

Note that the principal functions corresponding to the spectral singularities are not the elements of the Hilbert space. Also, the spectral singularities belong to the continuous spectrum and are not the eigenvalues. However, the spectral singularities play a certain critical role in the spectral analysis of operators. Their existence is accompanied by specific phenomenon which are new in the sense that they do not occur either in the spectral analysis of selfadjoint or normal operators.

The spectral singularities of the Sturm-Liouville operators with the general boundary conditions was investigated in detail by Krall [12], [13]. Some problems of spectral theory of differential equations and operators with spectral singularities were also studied in [14][19].

Let $S$ be a n-dimensional $(n<\infty)$ Euclidian space and we denote by $L^{2}(\mathbb{R}, S)$ the Hilbert space of vector-valued functions with values in $S$ and the norm

$$
\|f\|^{2}:=\int_{-\infty}^{\infty}\|f(x)\|_{S}^{2} d x .
$$

Let $L$ denote the operator generated in $L^{2}(\mathbb{R}, S)$ by the matrix Schrödinger equation

$$
-y^{\prime \prime}+Q(x) y=\lambda^{2} y \quad,-\infty<x<\infty
$$

where $Q$ is a non-self adjoint matrix-valued potential function $\left(Q \neq Q^{*}\right)$ and $\lambda$ is a spectral parameter. It is clear that, the operator $L$ is non-selfadjoint. $L$ is called the matrix Schrödinger operator.

In this paper, we investigate asymptotics and analytical properties of Jost function of (1.1). We also obtain the resolvent of $L$. Later, we study the eigenvalues and the spectral singularities of $L$ using the analytic continuation and uniqueness theorems of analytic functions. Afterwards we prove that the equation (1.1) 
(i.e. the operator $L$ ) has a finite number of eigenvalues and spectral singularities and each of them is of finite multiplicity under the condition

$$
\sup _{-\infty<x<\infty}\{\exp (\epsilon \sqrt{|x|})\|Q(x)\|\}<\infty \quad, \quad \epsilon>0 .
$$

\section{Jost function}

Suppose the matrix function $Q$ satisfies

$$
\int_{-\infty}^{\infty}(1+|x|)\|Q(x)\| d x<\infty .
$$

We introduce the notation

$$
\begin{array}{ll}
\eta^{+}(x)=\int_{x}^{\infty}\|Q(t)\| d t, & \eta_{1}^{+}(x)=\int_{x}^{\infty} \eta^{+}(t) d t, \\
\eta^{-}(x)=\int_{-\infty}^{x}\|Q(t)\| d t, & \eta_{1}^{-}(x)=\int_{-\infty}^{x} \eta^{-}(t) d t .
\end{array}
$$

Let $E^{+}(x, \lambda)$ and $F^{-}(x, \lambda)$ denote the solutions of (1.1) subject to the conditions

$$
\lim _{x \rightarrow \infty} E^{+}(x, \lambda) e^{-i \lambda x}=I, \quad \lambda \in \overline{\mathbb{C}}_{+}:=\{\lambda: \lambda \in \mathbb{C}, \operatorname{Im} \lambda \geq 0\},
$$

and

$$
\lim _{x \rightarrow-\infty} F^{-}(x, \lambda) e^{i \lambda x}=I, \quad \lambda \in \overline{\mathbb{C}}_{+},
$$

respectively, where $I$ denotes the identity matrix in $S$. Under the condition (2.1) the solution $E^{+}(x, \lambda)$ has the following integral representation [20]

$$
E^{+}(x, \lambda)=e^{i \lambda x} I+\int_{x}^{\infty} K^{+}(x, t) e^{i \lambda t} d t, \quad \lambda \in \overline{\mathbb{C}}_{+} .
$$

We also denote the solution of the equation

$$
-z^{\prime \prime}+z Q(x)=\lambda^{2} z \quad,-\infty<x<\infty,
$$

subject to the condition

$$
\lim _{x \rightarrow-\infty} z^{-}(x, \lambda) e^{i \lambda x}=I, \quad \lambda \in \overline{\mathbb{C}}_{+}
$$

by $E^{-}(x, \lambda)$.

Under the conditon (2.1), the solution $E^{-}(x, \lambda)$ has the similar integral representation

$$
E^{-}(x, \lambda)=e^{-i \lambda x} I+\int_{-\infty}^{x} K^{-}(x, t) e^{-i \lambda t} d t, \quad \lambda \in \overline{\mathbb{C}}_{+}
$$

where the matrix-functions $K^{ \pm}(x, t)$ are differentiable with respect to $x$ and $t$ and satisfies

$$
\begin{aligned}
& \left\|K^{ \pm}(x, t)\right\| \leq \frac{1}{2} \eta^{ \pm}\left(\frac{x+t}{2}\right) \exp \left\{\eta_{1}^{ \pm}(x)-\eta_{1}^{ \pm}\left(\frac{x+t}{2}\right)\right\}, \\
& \left\|K_{x}^{ \pm}(x, t) \mp \frac{1}{4} Q\left(\frac{x+t}{2}\right)\right\| \leq \frac{1}{2} \eta_{1}^{ \pm}(x) \eta^{ \pm}\left(\frac{x+t}{2}\right) \exp \eta_{1}^{ \pm}(x),
\end{aligned}
$$


1010

$$
\left\|K_{t}^{ \pm}(x, t) \mp \frac{1}{4} Q\left(\frac{x+t}{2}\right)\right\| \leq \frac{1}{2} \eta_{1}^{ \pm}(t) \eta^{ \pm}\left(\frac{x+t}{2}\right) \exp \eta_{1}^{ \pm}(t)
$$

The matrix-functions $E^{+}(x, \lambda)$ and $E^{-}(x, \lambda)$ are analytic with respect to $\lambda$ in $\mathbb{C}_{+}:=\{\lambda: \lambda \in \mathbb{C}, \operatorname{Im} \lambda>0\}$ and continuous up to the real axis.

Now, let us introduce

$$
D(\lambda):=W\left[E^{-}(x, \lambda), E^{+}(x, \lambda)\right], \quad \lambda \in \overline{\mathbb{C}}_{+},
$$

where $W\left[E^{-}(x, \lambda), E^{+}(x, \lambda)\right]$ is the Wronskian of the solutions of $E^{-}(x, \lambda)$ and $E^{+}(x, \lambda)$. The function $D$ is called Jost function of (1.1). Note that Jost function is analytic in $\mathbb{C}_{+}$ and continuous on the real axis.

Theorem 2.1. The function $D$ satisfies

$$
D(\lambda)=2 i \lambda I-2 K^{+}(0,0)-2 K^{-}(0,0)+\int_{0}^{\infty} f(t) e^{i \lambda t} d t
$$

where

$$
\begin{aligned}
f(t) & =K_{x}^{+}(0, t)-K_{x}^{-}(0,-t)-K_{t}^{+}(0, t)+K_{t}^{-}(0,-t)-K^{-}(0,0) K^{+}(0, t) \\
& -K^{+}(0,0) K^{-}(0,-t)+K^{-}(0,-t) * K_{x}^{+}(0, t)+K_{x}^{-}(0,-t) * K^{+}(0, t)
\end{aligned}
$$

in which $(*)$ is the convolution operation and $f \in L_{1}(\mathbb{R}, S)$.

Proof. By the definition of the Wronskian of the solutions $E^{-}(x, \lambda)$ and $E^{+}(x, \lambda)$ we have

$$
D(\lambda)=E^{-}(0, \lambda) E_{x}^{+}(0, \lambda)-E_{x}^{-}(0, \lambda) E^{+}(0, \lambda) .
$$

Using the integral represantations of $E^{-}(x, \lambda)$ and $E^{+}(x, \lambda)$ we obtain $(2.5)$. It follows from $(2.2)-(2.4)$ that $f \in L_{1}(\mathbb{R}, S)$.

Theorem 2.2. The following asymptotics hold

$$
\begin{aligned}
& D(\lambda)=2 i \lambda I-2 K^{+}(0,0)-2 K^{-}(0,0)+o(1) \quad, \lambda \in \overline{\mathbb{C}}_{+}, \quad|\lambda| \rightarrow \infty, \\
& D(\lambda)=2 i \lambda I+O(1) \quad \lambda \in \overline{\mathbb{C}}_{+}, \quad|\lambda| \rightarrow \infty .
\end{aligned}
$$

Proof. a) Let $\lambda \in \mathbb{R}$. By the Riemann-Lebesgue lemma for the Fourier transforms we get that [21]

$$
\int_{0}^{\infty} f(t) e^{i \lambda t} d t=o(1) \quad, \quad \lambda \in \mathbb{R}, \quad|\lambda| \rightarrow \pm \infty .
$$

b) Let $\lambda \in \mathbb{C}_{+}$. In this case, by the Lebesgue theorem we obtain that [21]

$$
\int_{0}^{\infty} f(t) e^{i \lambda t} d t=o(1) \quad, \quad \lambda \in \mathbb{C}_{+},|\lambda| \rightarrow \infty .
$$

It follows from (2.8) and (2.9) that

$$
\int_{0}^{\infty} f(t) e^{i \lambda t} d t=o(1) \quad, \quad \lambda \in \overline{\mathbb{C}}_{+},|\lambda| \rightarrow \infty .
$$

From (2.5) and (2.10) we have (2.6). In a similar way we may also prove (2.7). 


\section{Eigenvalues and Spectral Singularities of L}

Let us suppose that

$$
G(\lambda):=\operatorname{det} D(\lambda)
$$

Also, $\sigma_{d}(L)$ and $\sigma_{s s}(L)$ will denote the eigenvalues and spectral singularities of $L$, respectively. By the definition of eigenvalues and spectral singularities of differential operators we can write [7], [10]

$$
\begin{aligned}
& \sigma_{d}(L)=\left\{z: z=\lambda^{2}, \lambda \in \mathbb{C}_{+}, G(\lambda)=0\right\} \\
& \sigma_{s s}(L)=\left\{z: z=\lambda^{2}, \lambda \in \mathbb{R} \backslash\{0\}, G(\lambda)=0\right\} .
\end{aligned}
$$

Definition 3.1. The multiplicity of a zero of $G$ in $\overline{\mathbb{C}}_{+}$is defined as the multiplicity of the corresponding eigenvalue and spectral singularity of $L$.

In order to investigate the quantitative properties of the eigenvalues and the spectral singularities of $L$, we need to discuss the quantitative properties of the zeros of $G$ in $\overline{\mathbb{C}}_{+}$.

Let $M_{1}$ denotes the zeros of the function $G$ in $\mathbb{C}_{+}$and $M_{2}$ denotes the zeros of the function $G$ on the real axis. Therefore, using (3.2) and (3.3) we obtain

$$
\begin{aligned}
& \sigma_{d}(L)=\left\{z: z=\lambda^{2}, \lambda \in M_{1}\right\} \\
& \sigma_{s s}(L)=\left\{z: z=\lambda^{2}, \lambda \in M_{2}\right\} \backslash\{0\}
\end{aligned}
$$

Lemma 3.2. (i) The set $M_{1}$ is bounded and has at most countable number of elements and its limit points can lie only in a bounded subinterval of the real axis.

(ii) The set $M_{2}$ is compact and its Lebesque measure is zero.

Proof. From (2.7), we can obtain

$$
\left\|K^{ \pm}(x, t)\right\| \leq c \eta^{ \pm}\left(\frac{x+t}{2}\right)
$$

where $c>0$ is a constant. Using (3.1) and (3.6), we get that the function $G$ is analytic in $\mathbb{C}_{+}$, continuous on the real axis and satisfies the following

$$
G(\lambda)=2 i \lambda+O(1), \lambda \in \overline{\mathbb{C}}_{+},|\lambda| \rightarrow \infty
$$

Equation (3.7) shows that the sets $M_{1}$ and $M_{2}$ are bounded. Since $D(\lambda)$ is analytic in $\mathbb{C}_{+}$, then the set $M_{1}$ has at most countable number of elements. By (3.7) and the boundary value uniqueness theorem of analytic functions, we get that the set $M_{2}$ is closed and $\mu\left(M_{2}\right)=0$, where $\mu\left(M_{2}\right)$ denote Lebesgue measure of the set $M_{2}$ [22].

From (3.4),(3.5) and Lemma 3.2 we obtain the following theorem.

Theorem 3.3. Under the condition (2.1)

(i) The set of eigenvalues of $L$ is bounded, is no more than countable and its limit points can lie only in a bounded subinterval of the positive semiaxis.

(ii) The set of spectral singularities of $L$ is bounded and $\mu\left(\sigma_{s s}(L)\right)=0$.

Now, let us assume that, for some $\epsilon>0$,

$$
\int_{-\infty}^{\infty} \exp (\epsilon|x|)\|Q(x)\| d x<\infty .
$$

From $(2.2)-(2.4)$, we get the following

$$
\left\|K^{ \pm}(x, t)\right\|,\left\|K_{x}^{ \pm}(x, t)\right\|,\left\|K_{t}^{ \pm}(x, t)\right\| \leq c \exp \left[-\epsilon\left(\frac{x+t}{2}\right)\right]
$$


where $c>0$ is a constant. Also under the condition (3.8) we have

(3.10) $\quad\|f(t)\| \leq c e^{-\frac{\epsilon}{2}|t|}$

by $(3.9)$.

Theorem 3.4. Under the condition (3.8), the operator $L$ has a finite number of eigenvalues and spectral singularities and each of them is of finite multiplicity.

Proof. By using (3.9) and (3.10) we observe that the function $G$ has analytic continuation to the half plane $\operatorname{Im} \lambda>-\frac{\epsilon}{2}$. So, the limit points of zeros of $G$ can not lie in $\mathbb{R}$. Using Lemma 3.2, we get that the bounded sets $M_{1}$ and $M_{2}$ have a finite number of elements. Since $G$ is analytic for $\operatorname{Im} \lambda>-\frac{\epsilon}{2}$, we obtain that all zeros of $G$ in $\overline{\mathbb{C}}_{+}$have a finite multiplicity. So that the sets $\sigma_{d}(L)$ and $\sigma_{s s}(L)$ have a finite number of element with a finite multiplicity.

Now, let us suppose that

$$
\int_{-\infty}^{\infty} \exp (\epsilon \sqrt{|x|})\|Q(x)\| d x<\infty \quad, \quad \epsilon>0
$$

which is weaker than (3.8).

It is evident that under the condition (3.11), the function $G$ is analytic in $\mathbb{C}_{+}$and infinite differentiable on the real axis.

Let us denote the sets of all limit points of $M_{1}$ and $M_{2}$ by $M_{3}$ and $M_{4}$ respectively, and the set of all zeros of $G$ with infinite multiplicity in $\overline{\mathbb{C}}_{+}$by $M_{5}$. It is obvious from the uniqueness theorem of the analytic functions that

$$
M_{3} \subset M_{2}, M_{4} \subset M_{2}, M_{5} \subset M_{2}, M_{3} \subset M_{5}, M_{4} \subset M_{5}
$$

and $\mu\left(M_{3}\right)=\mu\left(M_{4}\right)=0[22]$.

Lemma 3.5. Under the condition (3.11), the function $G$ and its derivatives provide the following inequality:

$$
\left|G^{(n)}(\lambda)\right| \leq A_{n} \quad, \quad n=1,2, \ldots, \quad \operatorname{Im} \lambda>0
$$

where

$$
\begin{aligned}
& A_{1}=2+c 2^{2} \int_{0}^{\infty} t e^{-\frac{\epsilon}{2} \sqrt{t}} d t \\
& A_{n}=c 2^{n+1} \int_{0}^{\infty} t^{n} e^{-\frac{\epsilon}{2} \sqrt{t}} d t, \quad n=2,3, \ldots
\end{aligned}
$$

are constants. In addition, for all $n \in \mathbb{N}$

$$
A_{n} \leq B b^{n} n ! n^{n}
$$

holds where $B, b$ are constants.

Proof. We easily get the proof of the Lemma using (2.5) and (3.1).

Theorem 3.6. If (3.11) holds, then $M_{5}=\varnothing$.

Proof. Using Lemma 3.2, for sufficiently large $T>0$, we get

$$
\left|\int_{-\infty}^{T} \frac{\ln |G(\lambda)|}{1+\lambda^{2}} d \lambda\right|<\infty, \quad\left|\int_{T}^{\infty} \frac{\ln |G(\lambda)|}{1+\lambda^{2}} d \lambda\right|<\infty
$$


Since $G(\lambda) \neq 0$, we obtain

$$
\int_{0}^{h} \ln H(s) d \mu\left(M_{5}, s\right)>-\infty
$$

by (3.12), (3.14) and Pavlov's Theorem, where $H(s)=\inf _{n} \frac{A_{n} s^{n}}{n !}, \mu\left(M_{5}, s\right)$ is the Lebesque measure of the s-neighbourhood of $M_{5}$ and $h>0$ is a constant [5]. Substituting (3.14) into the definition $H(s)$, we arrive at

$$
H(s) \leq B \inf _{n}\left\{b^{n} s^{n} n^{n}\right\} \leq B \exp \left\{-b^{-1} s^{-1} e^{-1}\right\}
$$

and

$$
\ln H(s) \leq-b^{-1} s^{-1} e^{-1}
$$

Consequently,

$$
\int_{0}^{h} \frac{1}{s} d \mu\left(M_{5}, s\right)<\infty
$$

holds by using (3.15) for arbitrary $s$, if and only if $\mu\left(M_{5}, s\right)=0$ or $M_{5}=\varnothing$.

Lemma 3.7. $G$ has a finite number of zeros with finite multiplicity in $\overline{\mathbb{C}}_{+}$.

Proof. Since $M_{3} \subset M_{5}$ and $M_{4} \subset M_{5}$, we get

$$
M_{3}=M_{4}=\varnothing \text {. }
$$

By using Lemma 3.2 and (3.16), we obtain the finiteness of the sets $M_{1}$ and $M_{2}$. Because of $M_{5}=\varnothing$, all of the zeros of the function $G$ have finite multiplicities.

From Lemma 3.7, we get the following theorem.

Theorem 3.8. The operator $L$ has a finite number of eigenvalues and spectral singularities and each of them is of finite multiplicity if condition (3.11) holds.

\section{References}

[1] V. Lakshmikantham, D. D. Bainov, P. S. Simeonov, Theory of Impulsive Differential Equations, Series in Modern Applied Mathematics, vol. 6 (World Scientific Publishing Co, Inc., Teaneck, NJ, 1989).

[2] A. Mostafazadeh, H. Mehri-Dehnavi, Spectral singularities, biorthonotmal systems and a two-parameter family of complex point interactions, J. Phys. A. Math. Theory, 42 (2009), $125303(27 \mathrm{pp})$

[3] A. Mostafazadeh, Spectral singularities of a general point interaction, J. Phys. A. Math. Theory, 44 (2011), 375302 (9 pp).

[4] A. Mostafazadeh, Optical spectral singularities as threshold resonances, Physical Review A, 83, 045801 (2011).

[5] A. Mostafazadeh, Resonance phenomenon related to spectral singularities, complex barrier potential, and resonating waveguides, Physical Review A, 80, 032711 (2009).

[6] A. Mostafazadeh, Spectral singularities of complex scattering potentials and infinite reflection and transmission coefficients at real energies, Physical Review Letters, 102 , 220402 (2009).

[7] M.A. Naimark, Investigation of the spectrum and the expansion in eigenfunctions of a nonselfadjoit operators of second order on a semi-axis. AMS Transl. (2), 16 (1960), 103-193.

[8] J.T. Schwartz, Some non-self adjoint operators, Comm. Pure Appl. Math., 13 (1960), 609639.

[9] B. Nagy, Operators with spectral singularities, J. Oper. Theory, 15 (1986), 2, 307-325. 
[10] V. E. Lyance, A differential operator with spectral singularities, I, II, AMS Transl. (2), 60 (1967), 185-225, 227-283.

[11] B.S. Pavlov, The non-selfadjoint Schrödinger operator, Topics in Math Phys. 1, (1967), $87-110$.

[12] A.M. Krall, The adjoint of a differential operator with integral boundary conditions, Proc. Amer. Math. Soc., 16 (1965), 738-742.

[13] A.M. Krall, A non-homogenous eigenfunction expansion, Trans. Amer. Soc., 117 (1965), 352-361.

[14] A.M. Krall, E. Bairamov and O. Cakar, Spectrum and spectral singularities of a quadratic pencil of a Schrödinger operator with a general boundary condition, J. Differential Equations, 151 (1999), 2, 252-267.

[15] A.M. Krall, E. Bairamov and O. Cakar, Spectral analysis of a non-selfadjoint discrete Schrödinger operators with spectral singularities, Math. Nachr., 231 (2001), 89-104.

[16] E. Bairamov, O. Cakar and A.M. Krall, An eigenfunction expansion for a quadratic pencil of a Schrödinger operator with spectral singularities, J. Differential Equations 151 (1999), 268-289.

[17] E. Bairamov and A.M. Krall, Dissipative operators generated by Sturm-Liouville differential expression in the Weyl limit circle case J. Math. Anal. Appl., 254 (2001), 178-190.

[18] E. Bairamov, O. Cakar and C. Yanık, Spectral singularities Klein-Gordon s-wave equation, Indian J. Pure Appl. Math., 32 (2001), 851-857.

[19] E. Bairamov and O. Karaman, Spectral singularities of Klein-Gordon s-wave equations with an integral boundary condition, Acta Math. Hungar., 97 (2002), 1-2, 121-131.

[20] Z.S. Agranovich, V.A. Marchenko, The Inverse Problem of Scattering Theory, Gordon and Breach, New York, 1965.

[21] A.N. Kolmogorov and S.V. Fomin, Introductory Real Analysis, Dover Publ., New York, 1975.

[22] E.P. Dolzhenko, Boundary value uniqueness theorems for analytic functions, Math. Notes, 25 (1979), 437-442. 